University of Nebraska - Lincoln

DigitalCommons@University of Nebraska - Lincoln

Papers in the Earth and Atmospheric Sciences Earth and Atmospheric Sciences, Department

$5-1997$

\title{
Average Steady Nonuniform Flow in Stratified Formations
}

Peter Indelman

Tel Aviv University

Vitaly A. Zlotnik

University of Nebraska-Lincoln, vzlotnik1@unl.edu

Follow this and additional works at: https://digitalcommons.unl.edu/geosciencefacpub

Part of the Earth Sciences Commons

Indelman, Peter and Zlotnik, Vitaly A., "Average Steady Nonuniform Flow in Stratified Formations" (1997). Papers in the Earth and Atmospheric Sciences. 158.

https://digitalcommons.unl.edu/geosciencefacpub/158

This Article is brought to you for free and open access by the Earth and Atmospheric Sciences, Department of at DigitalCommons@University of Nebraska - Lincoln. It has been accepted for inclusion in Papers in the Earth and Atmospheric Sciences by an authorized administrator of DigitalCommons@University of Nebraska - Lincoln. 


\title{
Average steady nonuniform flow in stratified formations
}

\author{
Peter Indelman \\ Faculty of Engineering, Tel Aviv University, Tel Aviv, Israel \\ Vitaly Zlotnik \\ Department of Geology, University of Nebraska, Lincoln
}

\begin{abstract}
A mathematical model of average nonuniform flow in heterogeneous stratified media is developed. The averaged Darcy's law is obtained by deriving the analytical expression of the effective conductivity tensor for media with small heterogeneities. The fundamental solution of the average flow equation (mean Green function) corresponding to the flow toward a single point source of deterministic discharge is derived. The mean head distribution is calculated for a fully penetrating well, for a finite length well in an infinite domain, and for a dipole well. The concept of equivalent conductivity is defined for use in identifying the formation conductivity properties. The procedure for identifying the mean conductivity, log conductivity variance, and vertical integral scale is suggested for the case of dipole flow.
\end{abstract}

\section{Introduction}

Natural aquifers reveal a wide spatial variability of their properties, which affects their flow and transport parameters. The effect of formation structure on the process characteristics has been recognized for a long time, and numerous approaches that account for the formation heterogeneities have been suggested. The objective of these approaches is to develop theories applicable to both identifying aquifer parameters and forecasting flow and transport processes.

Early theories modeled formation heterogeneity by regarding hydraulic conductivities in the "horizontal" and "vertical" directions as two deterministic constants. Numerous classic solutions have been derived in the framework of the model of the deterministic anisotropic formation [e.g., Muskat, 1937; Polubarinova-Kochina, 1962]. These solutions have been found useful in developing various methodologies for identifying the aquifer parameters, mostly by radial well flow field experiments [e.g., Hantush, 1964; Streltsova, 1988]. It is clear that these models are unable to account for spatial variability of the conductivity and the uncertainty in our knowledge of such variations.

During the last three decades a stochastic approach to modeling flow and transport has been developed by considering the conductivity in the framework of random spatial processes. The objective of the stochastic approach is to characterize the flow and transport in terms of statistical moments of the variables of interest. This avenue has led to creating a stochastic theory of flow and transport in heterogeneous media that is available in a large number of papers and several books [e.g., Shvidler, 1985; Dagan, 1989; Gelhar, 1993]. These results have served as the basis for development of various methodologies leading to identification of the formation structure and have been applied to several field transport experiments [e.g., Sudicky, 1986; Hess et al., 1992; Rehfeldt et al., 1992].

The majority of results obtained using the stochastic theory

Copyright 1997 by the American Geophysical Union.

Paper number 96WR03790.

0043-1397/97/96WR-03790\$09.00 of flow and transport are restricted by the assumption of uniformity of average flow. This prevents application of the concept of stochasticity in domains of nonuniformity of the mean flow, in particular, in the vicinity of wells. Previously, only very special aspects of nonuniform flow were considered [e.g., Shvidler, 1966, 1985; Matheron, 1967; Dagan, 1989]. It is only recently that the stochastic approach was generalized for nonuniform flows in both steady [Neuman and Orr, 1993; Indelman and Abramovich, 1994] and unsteady cases [Indelman, 1996]. This approach proceeded by deriving the averaged equations and analysis of the properties of mean flow. The aim of the present work is to apply the general results of these studies to well hydraulics in heterogeneous layered formations.

We restrict our study to steady flow processes described by the flow equations

$$
\mathbf{q}=-K(\mathbf{x}) \boldsymbol{\nabla} h \quad \boldsymbol{\nabla} \cdot \mathbf{q}=\phi(\mathbf{x})
$$

where $\mathbf{q}$ and $h$ are the specific discharge and the hydraulic head, respectively; $\phi(x)$ is the deterministic source function; and $K(\mathrm{x})$ is the random conductivity. The main result obtained by Indelman and Abramovich [1994] is an expression of the effective Darcy's law, which relates the mean specific discharge $\langle q\rangle$ and the mean head gradient $\langle\nabla h\rangle$ by a convolution-type dependence

$$
\left\langle q_{n}(\mathbf{x})\right\rangle=-\int d \overline{\mathbf{x}} K_{n m}^{\mathrm{eff}}(\mathbf{x}-\overline{\mathbf{x}}) \frac{\partial\langle h(\overline{\mathbf{x}})\rangle}{\partial \bar{x}_{m}}
$$

where the kernel of the integral operator $K_{n m}^{\text {eff }}(\mathbf{x})$ is a tensor of the second order and depends only on statistics of the conductivity. (The standard convention on the summation on any repeated index holds.) The kernel $K_{n m}^{\text {eff }}$ is determined by its Fourier Transform (FT) $\tilde{K}_{n m}^{\text {eff }}(\mathbf{k})=\int d \mathbf{x} K_{n m}^{\text {eff }}(\mathbf{x}) \exp (i \mathbf{k} \cdot \mathbf{x})$, which is called the effective conductivity tensor (ECT). In terms of the $\log$ conductivity, $Y=\ln K$, and at the first-order approximation in the $\log$ conductivity variance $\sigma_{Y}^{2}$, the ECT is given by

$$
\bar{K}_{n m}^{\mathrm{eff}}(\mathbf{k})=K_{A}\left[\delta_{n m}-\sigma_{\gamma}^{2} \tilde{g}_{n m}(\mathbf{k})\right]
$$


In (3) $K_{A}$ is the mean conductivity and the tensor $\tilde{g}$ is determined in terms of the spectrum (FT) $\tilde{\rho}_{Y}$ (k) of the log conductivity autocorrelation function $\rho_{Y}(\mathbf{k})$, as follows:

$$
\tilde{g}_{n m}(\mathbf{k})=\int \frac{d \mathbf{p}}{(2 \pi)^{d}} \frac{p_{n} p_{m}}{p^{2}} \tilde{\rho}_{Y}(\mathbf{k}-\mathbf{p})
$$

where $p=|\mathbf{p}|$ and $d=1,2,3$ is the dimensionality of the problem. The mean head satisfies the integrodifferential equations

$$
\frac{\partial}{\partial x_{n}} \int d \overline{\mathbf{x}} K_{n m}^{\mathrm{eff}}(\mathbf{x}-\overline{\mathbf{x}}) \frac{\partial\langle h(\overline{\mathbf{x}})\rangle}{\partial \bar{x}_{m}}=\phi(\mathbf{x})
$$

Equation (5) determines the mean head for any source function $\phi(x)$, for example, for any distribution of the sources and sinks in space. The fundamental solution $G(x)$ of (5), that is, a solution for a single source $\phi(x)=K_{A} \delta(x)$, is called the mean Green function. This function is completely determined by its FT as follows:

$$
\begin{gathered}
G(\mathbf{x})=\int \frac{d \mathbf{k}}{(2 \pi)^{d}} \tilde{G}(\mathbf{k}) \exp (-i \mathbf{k} \cdot \mathbf{x}) \\
\tilde{G}(\mathbf{k})=\frac{K_{A}}{\tilde{Y}(\mathbf{k}) k^{2}}
\end{gathered}
$$

where $\bar{Y}(\mathbf{k})$ is the normalized contraction of the ECT given by

$$
\begin{gathered}
\tilde{Y}(\mathbf{k})=\frac{k_{n} \bar{K}_{n m}^{\text {eff }}(\mathbf{k}) k_{m}}{k^{2}}=K_{A}\left[1-\sigma_{Y}^{2} \tilde{g}(\mathbf{k})\right] \\
\tilde{g}(\mathbf{k})=\frac{k_{n} \tilde{g}_{n m}(\mathbf{k}) k_{m}}{k^{2}}
\end{gathered}
$$

It follows from (2)-(7) that for an arbitrary distribution of the sources $\phi$ the average head is given by

$$
\langle h(\mathbf{x})\rangle=\frac{1}{K_{A}} \int d \overline{\mathbf{x}} G(\mathbf{x}-\overline{\mathbf{x}}) \phi(\overline{\mathbf{x}})
$$

for any statistics of the log conductivity.

The aforementioned results have been derived by Indelman and Abramovich [1994] for the boundary condition where the deterministic source function $\phi$ describing wells is given. The case of deterministic head is considered by Indelman et al. [1996].

Expressions (2)-(8) constitute the mathematical model of average steady state flow driven by a system of sources and sinks. These equations permit one to determine the average flow quantities (mean head, its gradient, and mean velocity field), provided the tensor $\bar{g}_{n m}(\mathbf{k})$ is known. This tensor was derived early for isotropic two- and three-dimensional cases and for exponential and Gaussian covariance functions [Indelman and Abramovich, 1994]. In the present study we investigate average flow in stratified formations. Although the layered structure represents a simplified model of natural heterogeneity, the properties of nonuniform average flow in such a medium are quite complicated.

The plan of the paper is as follows: Following the outlined procedure, we derive the analytical expressions for the effective conductivity tensor for layered structure and for exponential covariance in section 2 . The mean Green function is calculated in section 3. In section 4 we illustrate the application of the mean Green function by investigating the flows driven by a fully penetrating well, by a well of finite length in an infinite domain, and by a dipole well. Section 5 discusses the concept of equivalent conductivity and its application to identifying formation properties from field experiments. Discussion of the concept of equivalent conductivity is carried out for the case of dipole flow; its generalization for other flow configurations is straightforward.

\section{Effective Conductivity Tensor}

We consider flow in porous formations of stratified structure. Let $\left(x_{1}, x_{2}, x_{3}\right)$ be the Cartesian coordinate system with the plane $\left(x_{1}, x_{2}\right)$ parallel to the strata. The autocorrelation function of the $\log$ conductivity $\rho_{Y}$ between the two points $x$ and $y$ depends only on the lag of the "vertical" coordinates $\rho_{Y}(x-y)=\rho_{Y}\left(x_{3}-y_{3}\right)$. Its FT is given by

$$
\tilde{\rho}_{Y}(\mathbf{k})=(2 \pi)^{2} \delta\left(\mathbf{k}_{r}\right) \Phi_{Y}\left(k_{3}\right)
$$

where $\mathbf{k}_{\boldsymbol{r}}$ is the coordinate vector in Fourier space along the plane $\left(k_{1}, k_{2}\right)$, and $\Phi_{Y}$ is the one-dimensional FT of $\rho_{Y}$. Substituting (9) into (4) leads to

$$
\begin{array}{ll}
\bar{g}_{n m}(\mathbf{k})=\int_{-\infty}^{\infty} \frac{d p_{3}}{2 \pi} \frac{k_{n} k_{m}}{k_{r}^{2}+p_{3}^{2}} \Phi_{Y}\left(k_{3}-p_{3}\right) & n, m=1,2 \\
\bar{g}_{n 3}(\mathbf{k})=\int_{-\infty}^{\infty} \frac{d p_{3}}{2 \pi} \frac{k_{n} p_{3}}{k_{r}^{2}+p_{3}^{2}} \Phi_{Y}\left(k_{3}-p_{3}\right) & n=1,2
\end{array}
$$

$\tilde{g}_{33}(\mathbf{k})=\int_{-\infty}^{\infty} \frac{d p_{3}}{2 \pi} \frac{p_{3}^{2}}{k_{r}^{2}+p_{3}^{2}} \Phi_{Y}\left(k_{3}-p_{3}\right)$

Further, we adopt the exponential correlation model of the vertical stratification $\rho_{Y}=\exp \left(-\left|x_{3}-y_{3}\right| / I\right)$ where $I$ is the integral scale of the log conductivity heterogeneity. Substituting the FT of the autocorrelation function $\Phi_{Y}\left(k_{3}^{\prime}\right)=2 I /\left(1+k_{3}^{2}\right)$ into (10) and taking quadratures yield the following expressions of the tensor $\tilde{g}_{n m}$ :

$$
\begin{gathered}
\tilde{g}_{n m}\left(\mathbf{k}^{\prime}\right)=\frac{k_{n}^{\prime} k_{m}^{\prime}}{k_{r}^{\prime}} \frac{1+k_{r}^{\prime}}{\left(1+k_{r}^{\prime}\right)^{2}+k_{3}^{\prime 2}} \quad n, m=1,2 \\
\tilde{g}_{n 3}\left(\mathbf{k}^{\prime}\right)=\frac{k_{n}^{\prime} k_{3}^{\prime}}{\left(1+k_{r}^{\prime}\right)^{2}+k_{3}^{\prime 2}} \quad n=1,2 \\
\tilde{g}_{33}\left(\mathbf{k}^{\prime}\right)=1-\tilde{g}_{11}-\tilde{g}_{11}=1-\frac{k_{r}^{\prime}\left(1+k_{r}^{\prime}\right)}{\left(1+k_{r}^{\prime}\right)^{2}+k_{3}^{\prime 2}}
\end{gathered}
$$

where $\mathbf{k}^{\prime}=\mathbf{k} I$. The FT of the specific discharge is now expressed by a local relation $\left\langle\tilde{q}_{n}\right\rangle=-\bar{K}_{n m}^{\text {eff }}\left\langle\tilde{E}_{m}\right\rangle$ where $\langle\widetilde{\mathbf{E}}\rangle$ is the FT of the mean head gradient $\langle\mathbf{E}\rangle=\nabla\langle\mathbf{h}\rangle$ and the ECT $\tilde{K}_{n m}^{\text {eff }}$ in (3) and (11) is given by a full tensor; that is, its nondiagonal components $\widetilde{K}_{n m}^{\text {eff }} \neq 0$. However, the dependence of $\langle\overline{\mathbf{q}}\rangle$ on $\langle\overrightarrow{\mathbf{E}}\rangle$ can be simplified. Indeed, it was shown by Indelman and Abramovich [1994] that since $\langle\mathbf{E}(\mathbf{x})\rangle$ is a potential field, the ECT can not be defined uniquely. For example, the first component can be written as

$$
\begin{aligned}
\left\langle\tilde{\boldsymbol{q}}_{1}(\mathbf{k})\right\rangle= & -K_{A}\left\{\left\langle\widetilde{E}_{1}(\mathbf{k})\right\rangle-\sigma_{Y}^{2}\left[\tilde{g}_{11}(\mathbf{k})\left\langle\tilde{E}_{1}(\mathbf{k})\right\rangle\right.\right. \\
& \left.\left.+\tilde{g}_{12}(\mathbf{k})\left\langle\tilde{E}_{2}(\mathbf{k})\right\rangle+\tilde{g}_{13}(\mathbf{k})\left\langle\tilde{E}_{3}(\mathbf{k})\right\rangle\right]\right\} \\
= & i K_{A}\left\{k_{1}-\sigma_{Y}^{2}\left[\tilde{g}_{11}(\mathbf{k}) k_{1}+\tilde{g}_{12}(\mathbf{k}) k_{2}+\tilde{g}_{13}(\mathbf{k}) k_{3}\right]\right\}\langle h(\mathbf{k})\rangle \\
= & -K_{A}\left[1-\sigma_{Y}^{2} \tilde{g}_{1}(\mathbf{k})\right]\left\langle\tilde{E}_{1}(\mathbf{k})\right\rangle
\end{aligned}
$$


where the last step was obtained by using expressions (11) of $\tilde{g}_{n m}$ and $\tilde{g}_{1}=\tilde{g}_{1 m} k_{m} / k_{1}$. A similar procedure applied to $\left\langle\tilde{q}_{2}(\mathbf{k})\right\rangle$ and $\left\langle\tilde{q}_{3}(\mathbf{k})\right\rangle$ shows that the ECT has a diagonal structure. In Fourier space the average Darcy's law is now obtained as follows:

$$
\begin{gathered}
\left\langle\tilde{q}_{n}(\mathbf{k})\right\rangle=-\tilde{K}_{n}^{\text {eff }}(\mathbf{k})\left\langle\tilde{E}_{n}(\mathbf{k})\right\rangle \\
\tilde{K}_{n}^{\text {eff }}(\mathbf{k})=K_{A}\left[1-\sigma_{\mathbf{r}}^{2} \tilde{g}_{n}(\mathbf{k})\right]
\end{gathered}
$$

where

$$
\widetilde{g}_{n}\left(\mathbf{k}^{\prime}\right)=1-\frac{k_{r}^{\prime}+\delta_{n 1}+\delta_{n 2}}{k^{\prime 2}+1+2 k_{r}^{\prime}}
$$

with $\delta_{n m}$ being the Kronecker tensor.

In physical space the averaged Darcy's law relates the mean specific discharge to the mean head gradient in a nonlocal way:

$\left\langle q_{n}(\mathbf{x})\right\rangle=-K_{A} \int d \overline{\mathbf{x}}\left[\delta(\mathbf{x}-\overline{\mathbf{x}})-\sigma_{\mathrm{Y}}^{2} g_{n}(\mathbf{x}-\overline{\mathbf{x}})\right]\left\langle E_{n}(\overline{\mathbf{x}})\right\rangle$

where $K_{n}^{\text {eff }}$ is the inverse FT of $\bar{K}_{n}^{\text {eff }}(\mathbf{k})$ (no summation in (12) and (14)).

For isotropic media it has been shown that in general the directions of $\langle\mathbf{q}\rangle$ and $\langle\mathbf{E}\rangle$ do not coincide [Indelman, 1996]. However, if the mean flow is radial, the vectors $\langle\mathbf{q}\rangle$ and $\langle\mathbf{E}\rangle$ are collinear. For stratified media, because $g_{1}=g_{2} \neq g_{3}$, the direction of the mean discharge does not coincide with the direction of the mean head gradient, even for radial flow towards a single source.

It is easy to see that far from the well, that is, at the limit $k \rightarrow 0, \tilde{g}_{1}=\tilde{g}_{2}=0$, whereas $\tilde{g}_{3}=1$. It follows from (14) that $\tilde{K}_{n}^{\text {eff }}=K_{A}$ for $n=1,2$ and $\bar{K}_{3}^{\text {eff }}=K_{H}$ where $K_{H}$ is harmonic mean conductivity. These results are in agreement with the well-known results for uniform flow. In contrast, close to the well, that is, when $k \rightarrow \infty, \tilde{g}_{n} \rightarrow 1$, and therefore $\tilde{K}_{n}^{\text {eff }} \rightarrow K_{H}$ for any $n$, in agreement with the results of Shvidler [1966, 1985] and Dagan [1989] and in agreement with the general result of Indelman and Abramovich [1994].

\section{Mean Green Function}

We now proceed with deriving the fundamental solution of the average flow equation (5). This solution corresponds to the distribution of the mean head for flow toward a point sink of unit rate in heterogeneous media of unit mean conductivity and is known as the mean Green function. With the mean Green function determined, the mean head distribution for other system configurations is simply expressed by the superposition rule (8).

The mean Green function results from (6) and (7) as a sum of the Green function of the Laplace equation $G_{0}(x)=$ $1 / 4 \pi x$ and the first order in $\sigma_{Y}^{2}$ correction $G_{2}(x)$ :

$$
G(\mathbf{x})=G_{0}(x)+\sigma_{Y}^{2} G_{2}(\mathbf{x})
$$

where $G_{2}$ is determined by its FT $\tilde{g} / k^{2}$ as follows:

$$
G_{2}(\mathbf{x})=\int \frac{d \mathbf{k}}{(2 \pi)^{3}} \frac{\tilde{g}(\mathbf{k})}{k^{2}} \exp (-i \mathbf{k} \cdot \mathbf{x})
$$

The function $\tilde{g}(\mathbf{k})$ is calculated by substituting (13) in (7). It yields

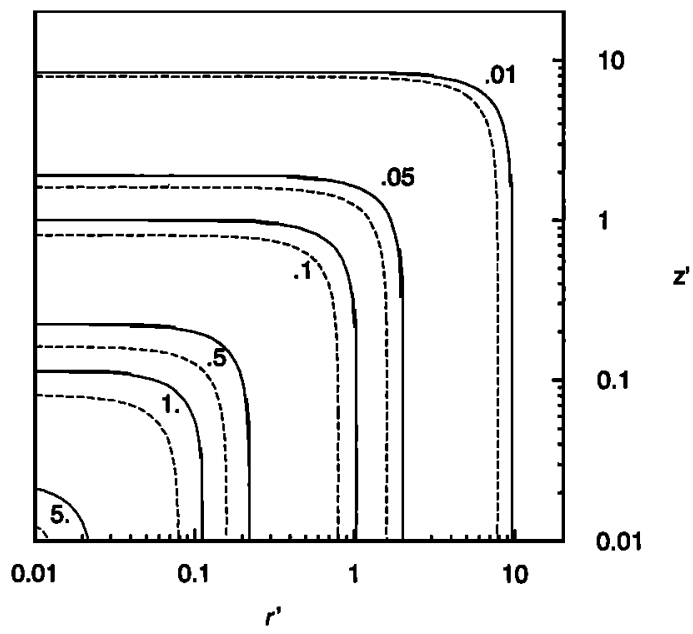

Figure 1. The mean head distribution $G\left(r^{\prime}, z^{\prime}\right)(15)$ in a stratified medium of $\sigma_{Y}^{2}=0.5$ (solid curve) and the head distribution $G_{0}\left(r^{\prime}, z^{\prime}\right)$ in a homogeneous medium for a flow toward a point source of unit rate.

$$
\tilde{g}\left(\mathbf{k}^{\prime}\right)=1-\frac{k_{r}^{\prime}}{k^{\prime 2}} \frac{k^{\prime 2}+k_{r}^{\prime}}{k^{\prime 2}+1+2 k_{r}^{\prime}}
$$

with $k^{\prime}=k I$.

Using this result the first order correction to the mean Green function is obtained as follows

$$
\begin{aligned}
G_{2}\left(r^{\prime}, z^{\prime}\right)= & \frac{G_{o}\left(x^{\prime}\right)}{2 \bar{I}}-\int_{0}^{\infty} \frac{d k_{r}^{\prime}}{8 \pi I} J_{0}\left(k_{r}^{\prime} r^{\prime}\right) e^{-k_{r}^{\prime}\left|z^{\prime}\right|} \frac{k_{r}^{\prime}}{1+2 k_{r}^{\prime}} \\
& \cdot\left[\left|z^{\prime}\right|-\frac{2 k_{r}^{\prime}}{1+2 k_{r}^{\prime}}\left(1+e^{-\left|z^{\prime}\right|}\right)\right]
\end{aligned}
$$

where $(r, z)$ are the cylindrical coordinates of the radial vector $\mathrm{x}, x=|\mathrm{x}|=\left(r^{2}+z^{2}\right)^{1 / 2}, \mathbf{x}^{\prime}=\mathrm{x} / I$, and $J_{0}$ is the Bessel function of the zero order. (Below, all spatial coordinates marked by a prime are scaled by the vertical integral scale $I$ ).

The integral in (18) was calculated numerically. Figure 1 compares the mean head (solid curves) for $\sigma_{Y}^{2}=0.5$ with the head in a homogeneous media (dashed curves). Note that the potential induced by the point source in the heterogeneous media is larger than that in the homogeneous media with similar $K_{A}$.

It is convenient to represent the mean Green function (15) as a product of the Green function for homogeneous media $G_{0}$ and function $\Psi\left(x^{\prime}\right)$, which comprises the effect of heterogeneity on the average head distribution

$$
\begin{gathered}
G\left(r^{\prime}, z^{\prime}\right)=\Psi\left(r^{\prime}, z^{\prime}\right) G_{0}\left(x^{\prime}\right) \\
\Psi\left(r^{\prime}, z^{\prime}\right)=1+\sigma_{Y}^{2} \psi\left(r^{\prime}, z^{\prime}\right) \\
\psi=G_{2} / G_{0}
\end{gathered}
$$

Note that unlike $Y(x)$ (the inverse FT of $\tilde{Y}(\mathbf{k})$ ), the function $\Psi(x)$ does not represent the intrinsic property of heterogeneous formation only, as it is defined for a flow toward a single point source and hence depends on $G_{0}$. Moreover, such a function, which relates the mean head to the head distribution in homogeneous media, can be defined for any flow configuration and we derive this function for several types of flow in next section. 


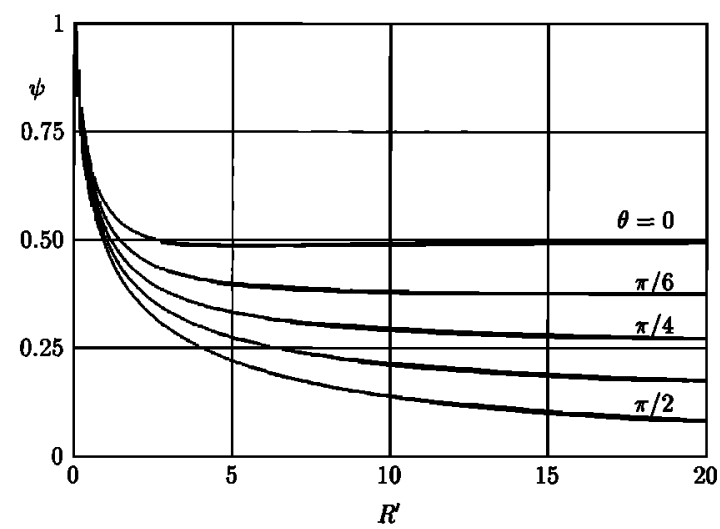

Figure 2. The first-order correction $\psi\left(r^{\prime}, z^{\prime}\right)$ (equations (18) and (19)) to the mean Green function as a function of $R^{\prime}=\left(r^{\prime 2}+z^{\prime 2}\right)^{1 / 2}$ for different directions $\theta=\arctan \left(z^{\prime} / r^{\prime}\right)$.

The first-order correction $\psi\left(r^{\prime}, z^{\prime}\right)$ is depicted in Figure 2 as a function of $R^{\prime}=\left(r^{\prime 2}+z^{\prime 2}\right)^{1 / 2}$ for different directions of $\theta=\arctan \left(z^{\prime} / r^{\prime}\right)$. The function $\psi$ decreases from 1 for $R^{\prime}=$ 0 to the asymptotic value for $R^{\prime} \rightarrow \infty$ depending on the angle $\theta$. Thus, for $r^{\prime}=0, \psi$ tends to zero with growth of $z$, whereas $\lim _{r^{\prime} \rightarrow \infty} \psi\left(r^{\prime}, 0\right)=1 / 2$. The order of decay with distance from source is more slow in the $z$ direction than in the $r$ direction. In the vicinity of the well, the mean flow is close to radial, and it does not depend on the direction $\theta$. This is manifested in unique value of $\psi(0,0)=1$. On the contrary, far from the well, the heterogeneity affects the mean streamlines in a different manner for different angles $\theta$.

\section{Applications of the Mean Green Function}

The aim of this section is to calculate the mean head distribution for several cases of interest with the aid of the fundamental solution of the average flow equation. The specific configuration of the flow system is prescribed by the source function $\phi(x)$ in the flow equation (5), whereas the mean head is determined by the principle of superposition (8) with $G$ given by (18) and (19). It follows from (8) and (15) that the mean head can be written in the form

$$
\langle h(\mathbf{x})\rangle=h_{0}(\mathbf{x})+\sigma_{Y}^{2}\left\langle h_{2}(\mathbf{x})\right\rangle
$$

where

$$
h_{0}(\mathbf{x})=\frac{1}{K_{A}} \int d \overline{\mathbf{x}} G_{0}(\bar{x}) \phi(\mathbf{x}-\overline{\mathbf{x}})
$$

is the head distribution in homogeneous deterministic media of conductivity $K_{A}$ and

$$
\left\langle h_{2}(\mathbf{x})\right\rangle=\frac{1}{K_{A}} \int d \overline{\mathbf{x}} G_{2}(\bar{r}, \bar{z}) \phi(\mathbf{x}-\overline{\mathbf{x}})
$$

is the first-order correction to $h_{0}$ with $G_{2}$ given by (18).

In the following we consider three cases of well flow driven by a fixed deterministic discharge, namely, flow to a fully penetrating well, to a well of finite length in infinite aquifer, and to dipole well. It is worthwhile to note that although the boundary condition of constant flux prevails in investigations, in many cases the correct condition on the well is that of constant head. Several attempts to solve the flow equations in homogenous media with a constant head condition [e.g., Muskat, 1937; Pol-
ubarinova-Kochina, 1962; Dagan, 1978] indicate the complicated structure of the solution. No solutions in a closed form are known at present [Haitjema, 1985; Cole and Zlotnik, 1994]. Analysis has shown that the difference between the two solutions occurs in a small vicinity of the screen edges. To simplify the calculations we neglect this effect and solve the equations subject to constant discharge.

\subsection{Flow to a Fully Penetrating Well}

For a fully penetrating well the source function is given by $\phi(\mathbf{x})=\kappa \delta(\mathbf{r})$ where $\mathbf{r}$ is a vectorial distance in the plane $\left(x_{1}, x_{2}\right)$ and $\kappa$ is the well discharge per unit screen length. Substituting $\phi$ into (21) and (22) yields the following expressions for mean head $\left\langle h^{(f)}(r)\right\rangle=h_{0}^{(f)}(r)+\sigma_{Y}^{2}\left\langle h_{2}^{(f)}(r)\right)$ :

$$
\begin{gathered}
h_{0}^{(f)}(r)=-\kappa \ln r /\left(2 \pi K_{A}\right) \\
\left\langle h_{2}^{(f)}(r)\right\rangle=\frac{\kappa}{K_{A}} \int_{-\infty}^{\infty} d \bar{z} G_{2}(r, \bar{z})
\end{gathered}
$$

Introducing (18) into (23) and calculating a quadrature one obtains

$$
\begin{aligned}
& \left\langle h^{(f)}(r)\right\rangle=\Psi^{(f)}(r) h_{0}^{(f)}(r) \\
& \Psi^{(f)}\left(r^{\prime}\right)=1+\sigma_{Y}^{2} \psi^{(f)}\left(r^{\prime}\right)
\end{aligned}
$$

where the first-order correction $\psi^{(f)}\left(r^{\prime}\right)$ is obtained as follows:

$$
\psi^{(\mathrm{f})}\left(r^{\prime}\right)=\frac{1}{\ln r^{\prime}} \int_{0}^{\infty} d k_{r}^{\prime} \frac{J_{0}\left(k_{r}^{\prime}\right)-J_{0}\left(k_{r}^{\prime} r^{\prime}\right)}{1+k_{r}^{\prime}}
$$

The correction $\psi^{(f)}$ is shown in Figure 3 as a function of $r^{\prime}$. Note the positivity and the limit values of $\psi^{(f)}: \lim _{r^{\prime} \rightarrow 0} \psi^{(f)}\left(r^{\prime}\right)$ $=1$ and $\lim _{r^{\prime} \rightarrow \infty} \psi^{(f)}\left(r^{\prime}\right)=0$. The mean flow is obviously radial along the plane of layering and does not depend on the $z$ coordinate. However, in each realization the flow has a threedimensional structure. Note that although it is far from the well that the mean head tends to its distribution in homogeneous media, the asymptotic limit is approached at distances of several orders of magnitude of the integral scale from the well.

Note that (24) corresponds to flow towards a well of given deterministic constant discharge along the well screen. The case of given deterministic head was studied by Indelman et al. [1996]. The solution (23)-(25) shows that similar to $h_{0}^{(f)}(r)$, the mean head is unbounded at large distances from the well. If

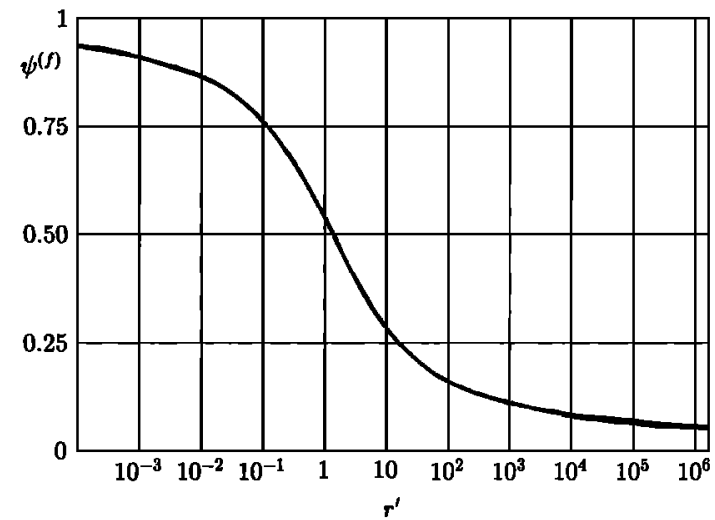

Figure 3. The first order correction $\psi^{(\mathrm{f})}\left(r^{\prime}\right)$ (equation (25)) to the mean head distribution for fully penetrating well. 
the mean head can be evaluated from the measurements at a specific distance from the well, one can rewrite the solution in bounded domain with the aid of the procedure used for homogeneous formations [e.g., Bear, 1979].

\subsection{A Well of Finite Length in an Infinite Domain}

For a well of finite screen length $2 \Delta$ and of constant discharge $Q$ in an infinite aquifer, the source function has a form

$\phi(\mathbf{r}, z)=2 \pi \kappa \delta(\mathbf{r})[H(z+\Delta)-H(z-\Delta)]$

with $H(z)$ being the Heaviside function and $\kappa=Q /(2 \Delta)$ being the well discharge per unit screen length.

Expression (26) was introduced into (21) and (22). After some quadratures the mean head $\left\langle h^{(\mathrm{p})}(r, z)\right\rangle=h_{0}^{(\mathrm{p})}(r, z)+$ $\sigma_{Y}^{2}\left\langle h_{2}^{(p)}(r, z)\right\rangle$ is obtained where

$$
h_{0}^{(\mathrm{p})}\left(r^{\prime}, z^{\prime}\right)=\frac{Q}{8 \pi K_{A} \Delta} \ln \frac{z_{-}^{\prime}-\left(r^{\prime 2}+z_{-}^{\prime 2}\right)^{1 / 2}}{z_{+}^{\prime}+\left(r^{\prime 2}+z_{+}^{\prime 2}\right)^{1 / 2}}
$$

is the well-known zero-order term [e.g., Bear, 1979] and

$$
\begin{aligned}
& \left\langle h_{2}^{(\mathrm{p})}\left(r^{\prime}, z^{\prime}\right)\right\rangle=\frac{1}{2} h_{0}^{(\mathrm{p})}\left(r^{\prime}, z^{\prime}\right)+\frac{Q}{16 \pi K_{A} \Delta} \\
& \cdot \int d k_{r}^{\prime} J_{0}\left(k_{r}^{\prime} r^{\prime}\right)\left[\Omega\left(z_{-}^{\prime}, k_{r}^{\prime}\right)-\Omega\left(z_{+}^{\prime}, k_{r}^{\prime}\right)\right]
\end{aligned}
$$

is the first-order correction. In (27) and (28), $z_{ \pm}^{\prime}=z^{\prime} \pm \Delta^{\prime}$, $\Delta^{\prime}=\Delta / I$, and

$$
\begin{gathered}
\Omega\left(z^{\prime}, k_{r}^{\prime}\right)=\operatorname{sign}\left(z^{\prime}\right)\left\{\left|z^{\prime}\right| \exp \left(-k_{r}^{\prime}\left|z^{\prime}\right|\right)\right. \\
-\frac{1-\exp \left(-k_{r}^{\prime}\left|z^{\prime}\right|\right)}{k_{r}^{\prime}}+\frac{2}{\left(1+2 k_{r}^{\prime}\right)^{2}} \\
\cdot\left[\left(1+3 k_{r}^{\prime}\right)\left[1-\exp \left(-k_{r}^{\prime}\left|z^{\prime}\right|\right)\right]\right. \\
\left.\left.+\frac{k_{r}^{\prime 2}}{1+k_{r}^{\prime}}\left[1-\exp \left(-\left(1+k_{r}^{\prime}\right)\left|z^{\prime}\right|\right)\right]\right]\right\}
\end{gathered}
$$

As in the case of the fully penetrating well, we introduce the function $\Psi^{(\mathrm{p})}\left(r^{\prime}, z^{\prime}\right)$ by the relationship

$$
\begin{gathered}
\left\langle h^{(\mathrm{p})}\left(r^{\prime}, z^{\prime}\right)\right\rangle=\Psi^{(\mathrm{p})}\left(r^{\prime}, z^{\prime}\right) h_{0}^{(\mathrm{p})}\left(r^{\prime}, z^{\prime}\right) \\
\Psi^{(\mathrm{p})}\left(r^{\prime}, z^{\prime}\right)=1+\sigma_{Y}^{2} \psi^{(\mathrm{p})}\left(r^{\prime}, z^{\prime}\right) \\
\left.\psi^{(\mathrm{p})}=\left\langle h_{2}^{(\mathrm{p})}\right\rangle / h_{0}^{(\mathrm{p})}\right\rangle
\end{gathered}
$$

The first-order correction $\psi^{(\mathrm{p})}\left(r^{\prime}, z^{\prime}\right)$ was calculated numerically. Figure 4 shows the isolines of $\psi^{(p)}$ for three values of $\Delta^{\prime}=\Delta / I=0.5,2.0$, and 5.0.

\subsection{Dipole Well}

During the last decade, interest has grown in so-called dipole flow, where the well consists of injection and production chambers separated in the middle by an impermeable packer with a pump. The water flows from the upper production chamber to the injection chamber through the pump and from injection chamber to the production chamber through the porous formation. Dipole flow has been studied in several recent publications [Kabala, 1993; MacDonald and Kitanidis, 1993; Zlotnik and Ledder, 1994, 1996]. In these investigations the aquifer is regarded as a deterministic anisotropic formation (a)

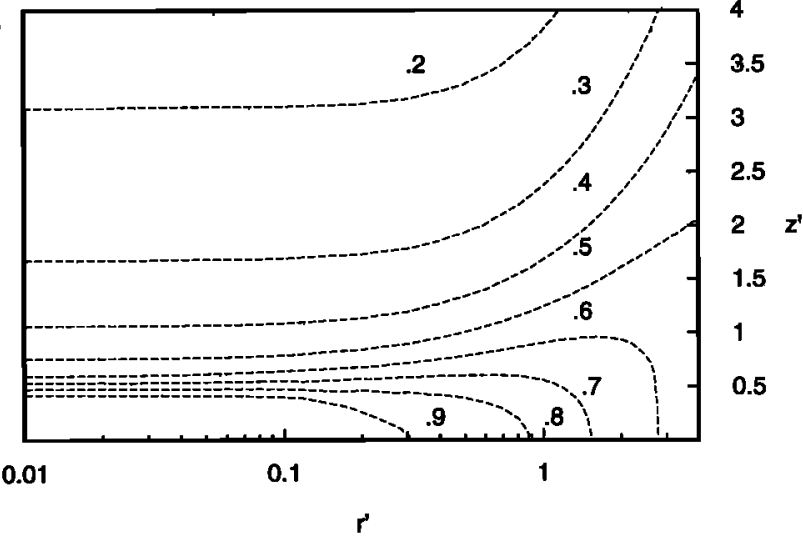

(b)

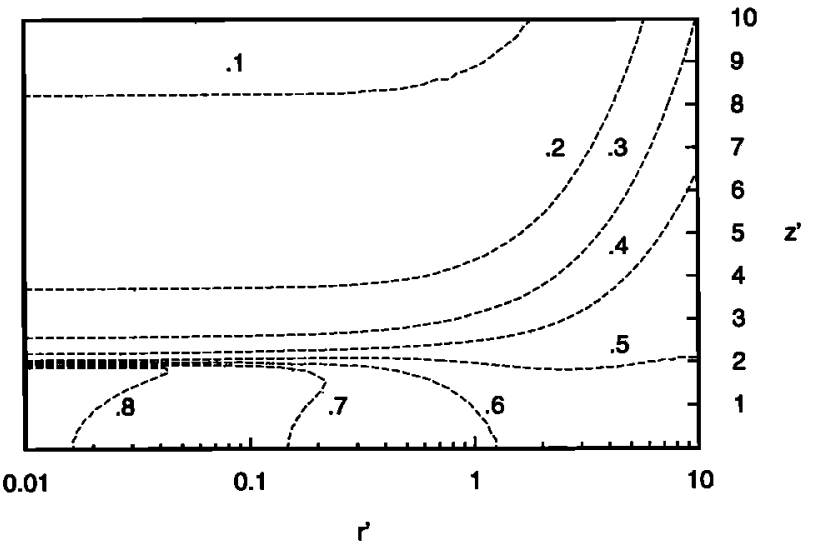

(c)

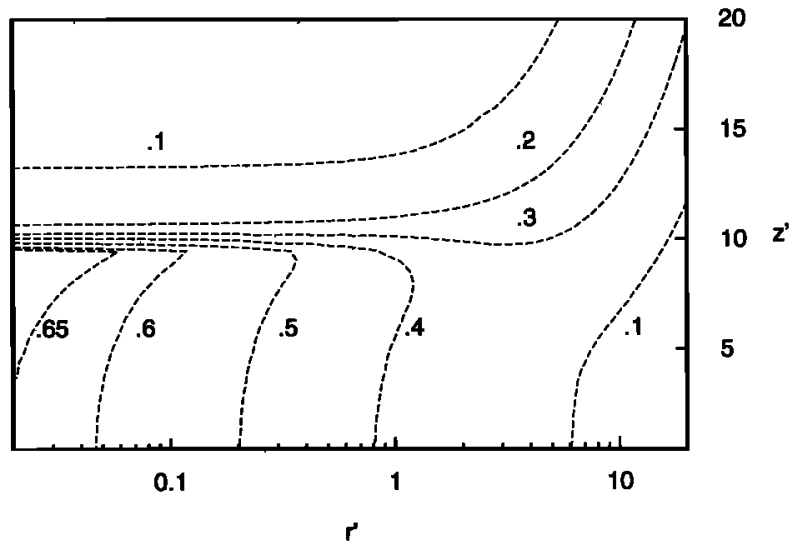

Figure 4. The first-order correction $\psi^{(\mathrm{p})}\left(r^{\prime}, z^{\prime}\right)$ (equations (28)-(30)) to the mean head distribution for finite length well and for (a) $\Delta^{\prime}=0.5$, (b) $\Delta^{\prime}=2$, and (c) $\Delta^{\prime}=10$.

with orthogonal axes of anisotropy (parallel and normal to the borehole). We aim to study dipole flow using a stochastic model of conductivity. Because of the relatively small size of the flow domain in the horizontal plane, it is reasonable to apply the stochastic stratified formation model to the analysis of dipole flow.

We consider a well of radius $r_{w}$ consisting of both upper and lower chambers, each of length $2 \Delta$. The distance between the centers of the two chambers is denoted as $2 L$. The water is pumped through the packer within the borehole from the upper chamber to the lower one with constant discharge $Q$. We restrict our consideration to the case when the upper (lower) chamber is placed far from the upper (lower) aquifer bound- 

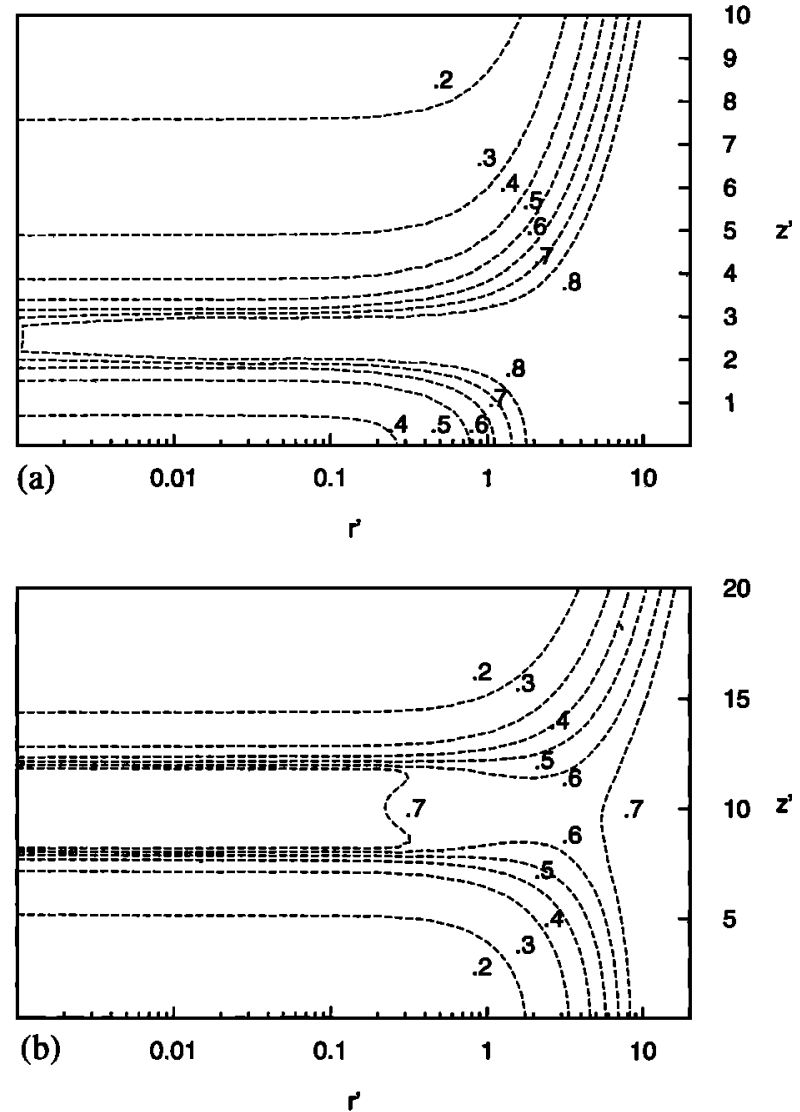

Figure 5. The first-order correction $\psi^{(\mathrm{d})}\left(r^{\prime}, z^{\prime}\right)$ (equations (32)-(34)) to the mean head distribution for dipole well, for $\Delta / L=0.2$ and (a) $L^{\prime}=2$ and (b) $L^{\prime}=10$.

aries, so that the flow domain can be considered infinite. We also assume that no natural gradient is applied.

Since the unsteady stage of the process is relatively short [Zlotnik and Ledder, 1994, 1996], we solve the steady state flow equations. The boundary condition on the well screen for both the upper and lower chambers is that of a given discharge per unit screen length $\kappa=Q /(2 \Delta)$, that is, $\kappa$ for $L-\Delta \leq z \leq$ $L+\Delta$ and $-\kappa$ for $-L-\Delta \leq z \leq-L+\Delta$. In practice, $r_{w} \ll L$, and this boundary condition is shifted to $r=0$.

Under these assumptions the distribution of the mean head $\left\langle h^{(d)}\right\rangle$ is simply expressed in terms of the mean head for a flow toward a finite length well in an infinite aquifer $\left\langle h^{(\mathrm{p})}(r, z)\right\rangle$, as follows:

$$
\left\langle h^{(\mathrm{d})}(r, z)\right\rangle=\left\langle h^{(\mathrm{p})}(r, z-L)\right\rangle-\left\langle h^{(\mathrm{p})}(r, z+L)\right\rangle
$$

Using the expansion $\left\langle h^{(\mathrm{d})}\right\rangle=h_{\mathrm{o}}^{(\mathrm{d})}+\sigma_{Y}^{2}\left\langle h_{2}^{(\mathrm{d})}\right\rangle$ and expressions (27) and (28) leads to the known zero-order head distribution [Zlotnik and Ledder, 1994, 1996]

$$
\begin{aligned}
h_{0}^{(\mathrm{d})}\left(r^{\prime}, z^{\prime}\right)= & \frac{Q}{8 \pi K_{A} \Delta}\left[\ln \left(\frac{z_{--}^{\prime}+\left(r^{\prime 2}+z_{--}^{\prime 2}\right)^{1 / 2}}{z_{-+}^{\prime}+\left(r^{\prime 2}+z_{-+}^{\prime 2}\right)^{1 / 2}}\right)\right. \\
& \left.-\ln \left(\frac{z_{+-}^{\prime}+\left(r^{\prime 2}+z_{+-}^{\prime 2}\right)^{1 / 2}}{z_{++}^{\prime}+\left(r^{\prime 2}+z_{++}^{\prime 2}\right)^{1 / 2}}\right)\right]
\end{aligned}
$$

and to the following first-order correction:

$$
\begin{aligned}
& \left\langle h_{2}^{\mathrm{d})}\left(r^{\prime}, z^{\prime}\right)\right\rangle=\frac{1}{2} h_{0}^{(\mathrm{d})}\left(r^{\prime}, z^{\prime}\right)+\frac{Q}{16 \pi K_{A} \Delta} \int d k_{r}^{\prime} J_{0}\left(k_{r}^{\prime} r^{\prime}\right) \\
& \cdot\left[\Omega\left(z_{--}^{\prime}, k_{r}^{\prime}\right)-\Omega\left(z_{-+}^{\prime}, k_{r}^{\prime}\right)-\Omega\left(z_{+-}^{\prime}, k_{r}^{\prime}\right)+\Omega\left(z_{++}^{\prime}, k_{r}^{\prime}\right)\right]
\end{aligned}
$$

where $\Omega\left(z^{\prime}, k_{r}^{\prime}\right)$ is defined by (29), $z_{ \pm \pm}^{\prime}=z^{\prime} \pm L^{\prime} \pm \Delta^{\prime}$ and $L^{\prime}=L / I$. The mean head is now given by

$$
\begin{gathered}
\left\langle h^{(\mathrm{d})}\left(r^{\prime}, z^{\prime}\right)\right\rangle=\Psi^{(\mathrm{d})}\left(r^{\prime}, z^{\prime}\right) h_{0}^{(\mathrm{d})}\left(r^{\prime}, z^{\prime}\right) \\
\Psi^{(\mathrm{d})}\left(r^{\prime}, z^{\prime}\right)=1+\sigma_{Y}^{2} \psi^{(\mathrm{d})}\left(r^{\prime}, z^{\prime}\right)
\end{gathered}
$$

with

$$
\psi^{(\mathrm{d})}\left(r^{\prime}, z^{\prime}\right)=\left\langle h_{2}^{(\mathrm{d})}\left(r^{\prime}, z^{\prime}\right)\right\rangle / h_{0}^{(\mathrm{d})}\left(r^{\prime}, z^{\prime}\right)
$$

The function $\psi^{(\mathrm{d})}\left(r^{\prime}, z^{\prime}\right)$ was calculated numerically, and its isolines are shown in Figure 5 for $\Delta / L=0.2$ and for $L^{\prime}=$ $L / I=2.0$ and 10.0 .

\subsection{Bounded Flows}

Since the average flow equations are linear in nature, one can determine the mean head distribution for bounded domains using, for example, the image technique. Consider flow towards a well of finite length $2 L$ with the center at $z=-z_{c}$ in a bounded formation $z<0$. If the boundary $z=0$ is impermeable, the mean head is obtained as a sum $\left\langle h^{(\mathrm{p})}\left(r, z+z_{c}\right)\right\rangle+$ $\left\langle h^{(p)}\left(r, z-z_{c}\right)\right)$. It should be emphasized, however, that the averaged equation (5) was derived for an infinite domain and is not correct close to the boundaries. Similar to the case of uniform flows [Rubin and Dagan, 1988, 1989], it is expected that the average equation is applicable for subdomains and wells that are far from the boundaries. The quantitative assessment of this statement is left for future study.

\section{The Equivalent Conductivity}

The results of the previous sections permit one to determine the mean head distribution for an arbitrary configuration of the system. Alternatively, these solutions can serve for identifying the properties of the formation. Thus, in the case of stratified media and a weak heterogeneity, the formation structure is determined by the arithmetic mean $K_{A}$, the logconductivity variance $\sigma_{Y}^{2}$, and the vertical integral scale $I$. The procedure for identifying the formation's properties on the basis of radial flow in heterogeneous media was developed recently by Indelman et al. [1996] for the boundary condition of given head. The procedure is based on the concept of equivalent conductivity, defined by Matheron [1967] as the conductivity of the fictitious homogeneous medium which produces a well discharge equal to the mean discharge in the actual formation. A similar concept can be defined for the case where flux is the given condition.

The definition of equivalent conductivity depends on the type of experiment and the measurements. We define equivalent conductivity for the dipole well flow, for which the measurable quantity is the chamber's head. With $r_{w}$ being the effective well radius we regard the measured head as the head in the middle of the chambers and at the well's wall (i.e., at points $\left.r=r_{w}, z= \pm L\right)$. The mean head at the same points is given by $\left\langle h^{(\mathrm{d})}\left(r_{w}, \pm L\right)\right\rangle=h_{0}^{(\mathrm{d})}\left(r_{w}, \pm L\right)+\sigma_{Y}^{2}\left\langle h_{2}^{(\mathrm{d})}\left(r_{w}, \pm L\right)\right\rangle$ with $h_{0}^{(\mathrm{d})}$ and $\left\langle h_{2}^{(\mathrm{d})}\right\rangle$ defined by (31) and (32). The equivalent conductivity for dipole flow is the conductivity of the fictitious homogeneous media for which the head drop between the centers of the upper and lower chambers is equal to the mean head drop. Note that unlike the effective conductivity, the equivalent conductivity depends on the specific problem studied and hence does not represent an intrinsic property of the medium. The advantage of the concept of equivalent conduc- 
tivity is that it represents a measurable quantity. Indeed, by carrying out the dipole flow experiment at different locations and (or) at different depths for fixed $\Delta$ and $L$, one can estimate the average head drop $\overline{\Delta h}$. The equivalent conductivity then results as follows:

$$
K^{\mathrm{eqv}}\left(r_{w}, L, \Delta\right)=\frac{2 K_{A} h_{0}^{(\mathrm{d})}\left(r_{w}, L\right)}{\bar{\Delta} h}
$$

where $h_{0}^{(\mathrm{d})}$ is given by (31) and the factor 2 indicates the head differential between the upper and lower chambers in uniform media.

On the other hand, the head drop in a homogeneous medium having conductivity $K^{\mathrm{eqv}}$ is equal to $2 h_{0}^{(\mathrm{d})}\left(r_{w}, L\right) K_{A} / K^{\mathrm{eqv}}$. Equating this head drop to the mean one $2\left\langle h^{(\mathrm{d})}\left(r_{w}, L\right)\right\rangle$ yields, according to derived equation (33),

$$
K^{\mathrm{eqv}}=K_{A} /\left[1+\sigma_{Y}^{2} \psi^{(\mathrm{d})}\left(r_{w}, L\right)\right]
$$

where $\psi^{(\mathrm{d})}$ given by (32)-(34) explicitly involves the dependence on $I$.

If $K^{\text {eqv }}$ is estimated from a series of experiments with various $L$ and $\Delta$ and from (35), the conductivity properties can be identified by fitting the measured $K^{\text {eqv }}$ to the calculated one (36). The latter depends on the conductivity properties through $K_{A}, \sigma_{Y}^{2}$, and $I$ and on the dipole geometric characteristics $r_{w}, \Delta$, and $L$ through the function $\psi^{(\mathrm{d})}\left(r_{w}, L\right)$. The function $\psi^{(\mathrm{d})}\left(r_{w}, L\right)$ is shown in Figure 6 as a function of $L^{\prime}=$ $L / I$ for $r_{w}^{\prime}=r_{w} / I=2$ and for several values of the ratio $\bar{\Delta}=\Delta / L$.

\section{Summary and Conclusions}

This study is devoted to developing a mathematical model for average flow in stratified formations and to applying this model to identifying the formation properties. The approach is based on the previously developed general model for average flow [Indelman and Abramovich, 1994; Indelman, 1996]. The assumption that the formation has a layered structure significantly simplifies calculations and leads to solutions in the form of simple quadratures.

Our first result is the effective conductivity tensor and the nonlocal average Darcy's law. We have shown that in the principal coordinate axes of local conductivity, the $n$th component of the average velocity depends only on the same component of the mean head gradient, that is, the effective conductivity tensor has a diagonal structure. However, the direction of the mean velocity deviates from that of the mean head gradient. We show that at distances from the source much larger than the integral scale of the log conductivity, the effective conductivity tensor approaches its value for uniform flow in stratified media, that is, the arithmetic and harmonic means. In contrast, close to the well the effective conductivity tensor tends to the harmonic mean.

The second result of the study is derivation of the fundamental solution of the average flow equation for the mean head, which is called the mean Green function. This solution corresponds to the mean head distribution for the flow toward a single point source and constitutes a basis for calculating solutions for any other flow configuration. Computation of the mean Green function requires one-dimensional integration. We have illustrated the behavior of the fundamental solution at different limits.

Using the mean Green function, one can calculate the mean

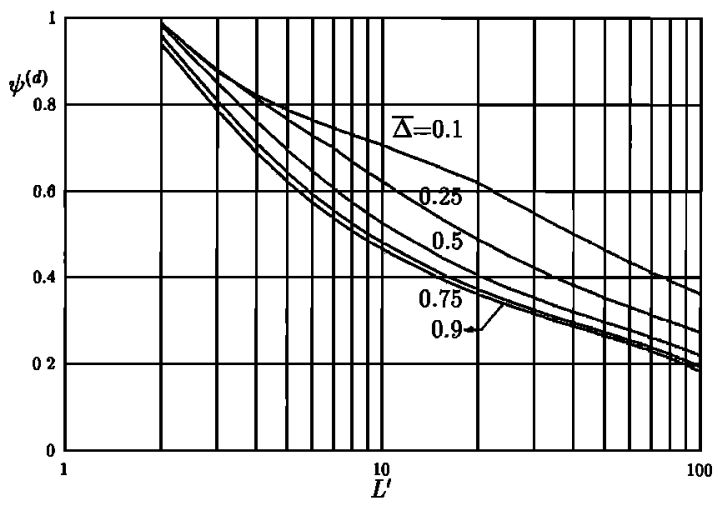

Figure 6. The first-order correction $\psi^{(\mathrm{d})}\left(r_{w}^{\prime}, L^{\prime}\right)$ to the equivalent conductivity for dipole well, for $r_{w}^{\prime}=2$ and for $\bar{\Delta}=$ $\Delta / L=0.1,0.25,0.5,0.75$, and 0.9 .

head distribution for specific distributions of sources and sinks. We have restricted our discussion to single-well flow and have considered the three most typical cases: the fully penetrating well, the finite length well in an infinite aquifer, and the dipole well. For each case we derive the correction function $\Psi\left(x^{\prime}\right)$, which should be used to multiply the head distribution in homogeneous media to adjust the latter to the mean head value. It is obvious that the mean head in any wells' system can easily be obtained from those considered in the paper. We have also discussed the solution of the flow problem in bounded aquifers.

Our third result is related to the problem of identification of the formation parameters. We show how one can define the so-called equivalent conductivity in order to identify the log conductivity statistics from measurements of the head. This procedure was developed previously for well flow with a given head [Indelman et al., 1996]. Here we define a similar approach for the case of a given well-pumping rate. Although a discussion of dipole flow is given, the approach is easily applied to any other flow configuration. The theoretical development permits one to identify conductivity statistical characteristics if the average mean head can be estimated from the measurement procedure. We hope to apply this procedure to dipole flow in the field in future work.

Acknowledgments. This work was partially supported by the Water Center of the University of Nebraska-Lincoln, Nebraska Research Initiative, and grant from the National Water Research Institute, United States. The authors wish to thank Carl McElwee and an anonymous reviewer for their helpful comments.

\section{References}

Bear, J., Hydraulics of Groundwater, McGraw-Hill, New York, 1979.

Cole, K., and V. Zlotnik, Modification of Dagan's method for slug and packer test interpretation, in Computational Methods in Water Resources $X$, vol. 1, edited by A. Peters et al., pp. 719-726, Kluwer Acad., Norwell, Mass., 1994.

Dagan, G., A note on packer, slug, and recovery tests in unconfined aquifers, Water Resour. Res., 14, 929-934, 1978.

Dagan, G., The generalization of Darcy's law for nonuniform flows, Water Resour. Res., 15, 1-7, 1979.

Dagan, G., Flow and Transport in Porous Formations, Springer-Verlag, New York, 1989.

Gelhar, L. W., Stochastic Subsurface Hydrology, Prentice-Hall, Englewood Cliffs, N. J., 1993.

Haitjema, H., Modeling three-dimensional flow in confined aquifers by 
superposition of both two- and three-dimensional analytic functions, Water Resour. Res., 21, 1557-1566, 1985.

Hantush, M. S., Hydraulics of wells, in Advances in Hydroscience, vol. 1, edited by W. T. Chow, pp. 281-432, Academic, San Diego, Calif., 1964.

Hess, K. M., S. H. Wolf, and M. A. Celia, Large-scale natural gradient tracer test in sand and gravel, Cape Cod, Massachusetts, 3, Hydraulic conductivity variability and calculated macrodispersivities, Water Resour. Res., 28, 2011-2027, 1992

Indelman, P., Averaging of unsteady flows in heterogeneous media of stationary conductivity, J. Fluid Mech., 310, 39-60, 1996.

Indelman, P., and B. Abramovich, Nonlocal properties of nonuniform averaged flows in heterogeneous media, Water Resour. Res., 30 , 3385-3393, 1994.

Indelman, P., A. Fiori, and G. Dagan, Steady flow toward wells in heterogeneous formations: Mean head and equivalent conductivity, Water Resour. Res., 32, 1975-1983, 1996.

Kabala, Z. J., Dipole flow test: A new single-borehole test for aquifer characterization, Water Resour. Res., 29, 99-107, 1993.

MacDonald, T. P., and P. K. Kitanidis, Modeling of the free surface of an unconfined aquifer near recirculation well, Ground Water, 31, 774-780, 1993.

Matheron, G., Elements Pour une Theorie des Milieux Poreux, Masson et Cie, Paris, 1967.

Muskat, M., The Flow of Homogeneous Fluids Through Porous Media, McGraw-Hill, New York, 1937.

Neuman, S. P., and S. Orr, Prediction of steady state flow in nonuniform geologic media by conditional moments: Exact nonlocal formalism, effective conductivities, and weak approximation, Water $R e$ sour. Res., 29, 341-364, 1993.

Polubarinova-Kochina, P. Y., Theory of Groundwater Movement, translation from Russian by R. J. M. de Wiest, Princeton Univ. Press, Princeton, N. J., 1962.
Rehfeldt, K. R., J. M. Boggs, and L. W. Gelhar, Field study of dispersion in a heterogeneous aquifer, 3 , Geostatistical analysis of hydraulic conductivity, Water Resour. Res., 28, 3309-3324, 1992.

Rubin, Y., and G. Dagan, Stochastic analysis of boundaries effects on head spatial variability in heterogeneous aquifers, 1 , Constant head boundaries, Water Resour. Res., 24, 1689-1697, 1988.

Rubin, Y., and G. Dagan, Stochastic analysis of boundaries effects on head spatial variability in heterogeneous aquifers, 2, Impervious boundary, Water Resour. Res., 25, 707-712, 1989.

Shvidler, M. I., The source-type solution of the problem of unsteady flow in random porous media, Izv. Akad. Nauk USSR Mekh. Zhidk. Gaza, 4, 137, 1966. (English translation, Fluid Dynamics, 4, 95-98, 1966.)

Shvidler, M. I., Stochastic Hydrodynamics of Porous Media (in Russian), Nedra, Moscow, 1985.

Streltsova, T. D., Well Testing in Heterogeneous Formations, John Wiley, New York, 1988.

Sudicky, E., A natural gradient experiment on solute transport in a sand aquifer: Spatial variability of hydraulic conductivity and its role in the dispersion process, Water Resour. Res., 22, 2069-2082, 1986.

Zlotnik, V., and G. Ledder, Effect of boundary conditions on dipole flow, in Computational Methods in Water Resources X, vol. 2, edited by A. Peters, et al., pp. 907-914, Kluwer Acad., Norwell, Mass., 1994.

Zlotnik, V., and G. Ledder, Theory of dipole flow in uniform anisotropic aquifers, Water Resour. Res., 32, 1119-1128, 1996.

P. Indelman, Faculty of Engineering, Tel Aviv University, P.O. Box 39040, Ramat Aviv, Tel Aviv, Israel. (e-mail: peter@eng.tau.ac.il)

V. Zlotnik, Department of Geology, University of Nebraska, Lincoln, NE 68508.

(Received September 23, 1996; accepted December 9, 1996.) 